\title{
Nutrición y actividad física en personas con discapacidad intelectual
}

\author{
Nutrition and physical activity in \\ people with intellectual disabilities
}

\begin{abstract}
People with intellectual disability (PID) (0.7-1.5\% of the population) have higher rates of obesity, since caloric values and nutritional status are deficient. We aimed to determine nutritional habits, analyze the effectiveness of nutritional education and improvement by introducing exercise and nutrition workshops, in a group with ID. Nutritional, anthropometric and clinical assessment was conducted in 19 patients. An ad hoc survey was designed in which exercise habits, medical and dietary history, record of 72 hours (including 2 weekdays, 1 weekend) and adherence to Mediterranean Diet data were collected. The workshops of exercise and nutrition counted with theoretical-practical explanation and games. The study population $(n=19)$, mean age 31 $( \pm 5.9)$ years, eleven men, eight women had an anthropometric assessment that showed $42 \%$ overweight and $16 \%$ obese. $11 \%$ had waist circumference of risk. None met the recommendations of daily exercise. $100 \%$ had inadequate nutritional intake. The intake and the prevalence of obesity in ID are inadequate. Nutritional education and physical exercise workshops are useful tools in this group, achieving significant benefits.

Keywords: Intellectual disability; food and nutrition education; obesity; exercise; habits.
\end{abstract}

\section{INTRODUCCIÓN}

Según la Asociación Americana para el Retraso Mental, la discapacidad intelectual (DI) se refiere a limitaciones sustanciales en el funcionamiento intelectual. Se caracteriza por un funcionamiento intelectual bajo con respecto a la media (1). La DI afecta aproximadamente $0,7 \%$ a 1,5\% de la población, en España supone más de 400.000 personas afectadas (2). Las personas con discapacidad intelectual (PDI) presentan mayores tasas de obesidad (doble de la población general), epilepsia (25 veces más) y trastornos mentales, así como de déficits sensoriales, problemas de movilidad, trastornos psicóticos y dolor bucal (2).

Las dificultades más comunes de las PDI respecto a la nutrición se centran en la calidad de la dieta, particularmente en los valores calóricos y el estado nutricional que parecen estar influidos por la habilidad de la persona para alimentarse por sí mismos (destreza motora, elección, conducta alimentaria, entre otras.). La deficiencia nutricional más común observada en PDI, es una insuficiencia general de nutrientes (malnutrición), seguida de problemas de habilidad para alimentarse,
Ismael San Mauro Belén García de Angulo Jesús Onrubia Diana Pina

Esther Fortúnez

Pablo Villacorta

Cristina Sanz

Paloma Galdine

$M^{a}$ Ángeles Bonilla

Elena Garicano Vilar

Centros de Investigación en Nutrición y Salud. Grupo de investigación. Madrid. España.

Dirigir la correspondencia a: Elena Garicano Vilar Centro de Investigación en Nutrición y Salud. Madrid. España.

Este trabajo fue recibido el 5 de Enero de 2016 y aceptado para ser publicado el 6 de Septiembre de 2016.

por lo que deben considerarse como un grupo vulnerable y de alto riesgo nutricional (1). Sin embargo, algunos autores han puesto de manifiesto que con una intervención basada en la educación nutricional, hábitos saludables y ejercicio físico, se puede mejorar esa situación y estado nutricional (3).

Esta población presenta una elevada prevalencia de obesidad, lo que se debería sobre todo a factores genéticos, ya que se produce una disminución del índice metabólico en reposo (4), y también a la falta de ejercicio físico y sedentarismo acompañados de la ingesta de gran cantidad de alimentos, ricos en azúcares, grasas y energía (Kcal) (5). Una solución podría ser la adquisición de unos hábitos dietéticos saludables desde la primera infancia (6).

Los jóvenes con DI a menudo experimentan un exceso de peso en mayor proporción que sus compañeros con desarrollo normal, aumentando el riesgo de una mayor masa corporal. Estas, incluyen una mayor utilización de medicamentos, síndromes asociados con la obesidad como el síndrome metabólico, y la posesión de hábitos alimentarios alterados relacionados con su discapacidad (7). 
La eficacia de la dietoterapia y la actividad física en situación de aumento de peso en PDI, depende considerablemente de los propios individuos, pero a pesar de ello el número de los que manejan con éxito bajar de peso aún no es satisfactorio ni suficiente. Investigadores observaron los casos de residentes en el Centro de Educación de Tarnow, Polonia y concluyeron que prevenir o tratar el exceso de peso en las PDI es extremadamente difícil, por todos los factores influyentes (8).

En el 2011, Heller T et al. (9) indicaron que al combinar programas de ejercicio físico para adultos con DI, con programas de educación en hábitos de salud más comprensibles, que incluyan información sobre ejercicio y nutrición, existían ciertas evidencias de mejoras en el peso.

Estas personas suelen tener problemas de nutrición, como déficits de micronutrientes o exceso de masa grasa y peso, así como bajo tono muscular. Pero, ¿estas condiciones son debidas a las patologías en sí o se ven expresadas cuando se dan unos hábitos alimentarios y de estilo de vida desfavorables? ¿Está socialmente extendida la sobreprotección y sobrecompensación a esta población, descuidando otros aspectos relevantes para su salud como la alimentación y el ejercicio físico? En ocasiones el entorno familiar ejerce como protector y se centra en el cumplimiento del tratamiento farmacológico, el progreso de habilidades psicomotoras, sociales y conductuales, descuidando otros aspectos básicos como su alimentación. Para contestar a estas preguntas se diseñó un estudio en el que se evaluaron los hábitos alimentarios y ejercicio de un grupo de 19 voluntarios con DI, en relación a su estado de salud, clínico y medidas antropométricas en Madrid (España), de la Fundación Síndrome de Down de Madrid (dentro del proyecto DOMUS, que integra a beneficiarios de dicha fundación a los que se les incluye en pisos tutelados para capacitarles en autonomía, reinserción laboral, etc.) y de la Asociación Avante3 de Leganés (Madrid).

El objetivo de esta investigación fue conocer los hábitos nutricionales de un grupo de personas con DI en Madrid, y analizar la eficacia de una intervención de educación nutricional y evaluar el posible efecto de mejora introduciendo talleres de ejercicio físico además de los talleres de nutrición entre ambos centros (AVANTE y DOMUS).

\section{SUJETOS Y METODOS}

Se realizó una valoración nutricional, antropométrica y clínica a 19 usuarios de la Fundación Síndrome de Down de Madrid $(n=8)$ y Avante3 de Madrid $(n=11)$. El muestreo fue incidental, en el cual los investigadores determinaron deliberadamente qué individuos formaron parte de la muestra en función de las asociaciones, tratando de escoger a los casos considerados más representativos de la población.

Todos los participantes y sus familias o tutores legales fueron informados, de forma escrita y oral por los monitores de las entidades participantes, del origen y finalidad del estudio, firmando una hoja de consentimiento informado, antes de comenzar el estudio, una vez entendieron y aceptaron las características del mismo. Para el estudio se diseñó una encuesta Ad Hoc en la que se recogieron datos sobre sus hábitos deportivos, historia clínica; además de la historia dietética de cada uno para conocer sus hábitos alimentarios y un registro alimentario (registro de 72 horas) donde se incluyeron 2 días laborales y 1 en fin de semana, y el cuestionario KidMed (10). Se revisaron las recomendaciones de objetivos nutricionales (macronutrientes y fibra) y de alimentación (cuestionario de calidad de dieta KidMed) para establecer la calidad de los hábitos encontrados entre los sujetos.
Estos datos fueron recogidos por dietistas-nutricionistas entrenados y formados, homogenizando con un protocolo de recogida de datos y monitorización del estudio.Para ello fue necesaria la ayuda del monitor que convivía con los sujetos en los pisos tutelados. Los datos recogidos fueron el peso, la talla (definida como la distancia entre el vértex y el plano de sustentación, medida con un estadiómetro SECA 216, rango 3,5 a $230 \mathrm{~cm}$, con una precisión de $1 \mathrm{~mm}$. Se realizó con el sujeto de pie, sin zapatos, completamente estirado, la cabeza se mantuvo en el plano horizontal al conducto auditivo externo (plano de Frankfurt). El porcentaje de grasa corporal, la masa libre de grasa $(\mathrm{Kg})$, el porcentaje de agua de cada individuo, el índice de masa corporal (IMC) (referido a lo establecido en la Sociedad Española para el Estudio de la Obesidad SEEDO o de acuerdo a la OMS) (11), perímetro de la cintura, frecuencia de consumo de alimentos, ejercicio, horas de sueño, presencia de enfermedades y tránsito intestinal.

Se revisaron los menús proporcionados desde las entidades colaboradoras o, en su caso, los menús de cada participante pautados por sus médicos de referencia. También se analizaron los registros dietéticos de 3 días para determinar la adecuación de su alimentación para sus necesidades y detectar los posibles hábitos o actitudes no saludables susceptibles de ser corregidos. Los datos dietéticos fueron analizados con el software DIAL @, con el que se pudo valorar la ingesta de nutrientes (hidratos de carbono, grasas, proteínas, colesterol y fibra dietética) e ingesta de energía (Kcal). Para la valoración antropométrica se usó bioimpedancia eléctrica tetrapolar, monofrecuencia $(50 \mathrm{kHz})$ modelo TANITA BC-601 con precisión de $100 \mathrm{~g}$ (rango 0,1-130 Kg) para ello se estandarizó un protocolo para la medición que consistía en: 1 - Ayuno previo de 3 horas como mínimo. 2 - Haber orinado antes de realizar la medición. 3 - No haber ingerido café, té o mate, ni haber realizado actividad física desde 12 horas antes de tomar la medición. 4 - No haber ingerido bebidas alcohólicas ni diuréticos desde 24 horas antes de realizar la impedancia. Para medir el perímetro de cintura se usaron cintas métricas de material flexible e inextensible (rango 0-150 cm), esta medición corresponde al menor contorno del abdomen y se encuentra en el punto medio entre el borde costal y la cresta iliaca. Se siguió el criterio marcado por otros autores previamente para definir el riesgo cardiovascular referido por el perímetro de la cintura, el cual sobre $88 \mathrm{~cm}$ para mujeres y 102 para hombres (12).

Los sujetos fueron distribuidos en dos grupos: el grupo DOMUS $(n=8)$ y el grupo AVANTE $(n=11)$. Todos los talleres contaron con una estructura de explicación teórica, práctica y juegos, para evaluar el entendimiento del contenido y los temas tratados fueron referentes a: grupos de alimentos, frecuencia de ingesta de alimentos, reparto de cargas calóricas a lo largo del día (5 comidas al día), desayuno, tamaño de raciones, hidratación, etiquetado, técnicas culinarias y hábitos saludables.

En cuanto a los talleres de deporte, que fueron introducidos sólo en el grupo de Avante3, se realizaron después de los talleres de nutrición por 2 profesionales de educación física adaptada, que seguían una estructura de explicación teórica, calentamiento, desarrollo de las actividades mediante pruebas, juegos, y estiramientos. Al finalizar, se llevaban a cabo ejercicios de relajación y de revisión constructiva de lo que se había trabajado para darle funcionalidad en la vida diaria.

Ambos talleres se dividieron en 5 sesiones, separadas por semanas (1 a la semana) y con una duración de $1 \mathrm{~h}$.

La intervención se midió en un punto temporal inicial (registro inicial), y un registro final a medio plazo, 3 meses 
después de finalizar el último taller en cada grupo. En ambos puntos temporales se realizaron los mismos cuestionarios y mediciones antropométricas, para estimar el cambio entre los puntos inicial-finales.

\section{ANÁLISIS ESTADÍSTICO}

Para el análisis estadístico de los datos se realizaron análisis descriptivos, presentando los resultados en medias, desviación estándar y porcentajes. Se utilizaron pruebas estadísticas paramétricas como el test de la prueba T y ANOVA para analizar las diferencias entre las medias de dos grupos de variables cuantitativas y prueba Chi-cuadrado para variables cualitativas no paramétricas, así como las prueba de rango de Wilconxon. Se comparó la diferencia inicial-final de todas las variables con la prueba $T$ para muestras relacionadas; y la prueba $T$ para muestras independientes, en el caso de la comparación inicialfinal del efecto de los grupos que realizaron o no realizaron ejercicio físico. Antes de cada test estadístico, se realizaron los test de normalidad al inicio, y entre grupos (test de KolmogorovSmirnoff) y test de homogeneidad (test de Levene). Se consideraron diferencias significativas con $p<0,05$, con un intervalo de confianza del 95\%. El análisis de los datos recolectados se procesó mediante el sistema SPSS (versión 21).

Para el cumplimiento de los objetivos nutricionales, se calculó el porcentaje (\%) de incremento o decremento del final con respecto al final, si por ejemplo incrementaron la puntuación del KidMed, que \% lo incrementó, quedando descrito.

\section{RESULTADOS}

La población estudiada $(n=19)$ presentaba diferentes discapacidades, con una edad media de $31( \pm 5,9)$ años, de los cuales once eran hombres (58\%) y ocho mujeres (42\%). Con respecto a la valoración antropométrica de los individuos, $5 \%$ presentaba bajo peso $(n=1), 37 \%$ normopeso $(n=7), 42 \%$ sobrepeso $(n=8)$ y $16 \%$ obesidad $(n=3)$. En cuanto al perímetro de la cintura, $11 \%$ de ellos estaban por sobre el perímetro de riesgo (cuadro 1). Los resultados de las encuestas sobre ejercicio físico se observó que ninguno cumplía con las recomendaciones de al menos 30 minutos diarios de ejercicio o actividad física o un equivalente en METs.

Se observó un desequilibrio en la ingesta de los individuos: la mayoría se encargaban del cocinado a diario, con alguna ayuda de personal de apoyo (monitores), reconociendo una cierta autonomía, que deriva en una ligera tendencia a técnicas culinarias sencillas, pero poco recomendadas, como por ejemplo las frituras.

Sólo 5\% $(n=1)$ cumplía con las recomendaciones diarias de fibra (situadas en 25-30 g/diarios). Con respecto a la grasa total hay diferencia entre la primera y segunda medición como se aprecia en la cuadro 2: en la primera encuesta al menos siete consumían más de $40 \%$ de grasa de la energía total diaria y en la segunda 10 de ellos consiguían reducir la ingesta de la misma. Sin embargo, las medias de ingesta de porcentaje energético en forma de grasa, fue muy parecido al inicio y al final (a los 3 meses de haber terminado el último taller de la intervención). Todo el exceso de grasa, que se ha comentado anteriormente, tiene relación con la ingesta energética y favorece que $58 \%$ de los participantes tenga sobrepeso u obesidad. En cuanto al consumo de fruta, se observó un descenso respecto al primer registro, ya que en este alcanzaban casi una ración y media $(1,35 \pm 0,87)$ diaria y en el análisis del segundo registro de 72 horas, casi no llegaba a una ración $(1,02 \pm 0,8)$ diaria.

Se observó una ligera mejora en el peso corporal, sin diferencias estadísticamente significativas $(p=0,99)$, ni tampoco en la grasa corporal $(p=0,8)$. No se observaron cambios significativos en la ingesta energética procedente de cada uno de los macronutrientes tras la intervención. Se observaron cambios significativos tras la intervención en el colesterol de la dieta $(p=0,004)$ y el perfil de calidad de la dieta medido mediante el cuestionario KidMed ( $p<0,001)$.

De los 11 candidatos con sobrepeso u obesidad, 7 de ellos (64\%) consiguieron bajar de peso, 1 se mantuvo (9\%) y otros 3 $(27 \%)$ subieron de peso. De los que perdieron peso $(n=7)$, dos (29\%) pasaron de obesidad (IMC>30) a sobrepeso (IMC entre 25 y 29,9), dos (28\%) se mantuvieron en sobrepeso, otros dos (29\%) bajaron de sobrepeso a normopeso y el último (14\%) se mantuvo en obesidad.

\section{TABLA 1}

Datos descriptivos iniciales.

Características de los participantes $(n=19)$

Frecuencia $(n)$

Sexo

Masculino

Femenino

11

8

Media (DE)

$31,1( \pm 5,9)$

Edad (años)

Peso (kg)

Talla (m)

IMC

Perímetro cintura $(\mathrm{cm})$

Masculino

Femenino
$71,4( \pm 20,0)$

$1,7( \pm 0,1)$

$25,8( \pm 4,6)$

$82,2( \pm 19,4)$

$87,4( \pm 14,3)$

$74,1( \pm 8,0)$
Porcentaje (\%)

58
42
Rango
$21-46$
$48,8-121,5$
$1,41-1,98$
$18,3-35,9$
$60,5-114,5$
$72,0-114,5$
$60,5-78,9$

Resumen de las características iniciales de los participantes sometidos a estudio, expresados como Media ( \pm Desviación Estándar) y agrupados en rangos o porcentajes. 
Al final de la intervención, 37\% aumentaron el ejercicio realizado a lo largo de la semana, 58\% mantuvo la actividad física que ya realizaba y sólo $5 \%$ diminuyó su ejercicio semanal.

Efecto de ejercicio físico programado en la intervención entre grupos:

Al separar a los participantes en dos grupos, el grupo DOMUS y el grupo AVANTE, se observó que de los beneficiarios del proyecto DOMUS mantuvieron su actividad física habitual, $50 \%(n=4)$, mientras que el otro $50 \%(n=4)$ la incrementó. Por otro lado, los de grupo AVANTE mantuvieron el ejercicio el $64 \%(n=7)$, lo aumentó el $27 \%(n=3)$ y lo disminuyó el 9\% $(n=1)$.

Se comparó entre ambos grupos la pérdida de peso producida y la disminución del porcentaje de grasa corporal, observándose que los participantes del grupo AVANTE redu- jeron de media $2,3 \mathrm{~kg}( \pm 2,6 \mathrm{~kg})$, frente a la ganancia media de $0,6 \mathrm{~kg}( \pm 2,9 \mathrm{~kg})$ de peso del grupo DOMUS. Tan sólo $27 \%$ $(n=3)$ de los pacientes de AVANTE subieron de peso, frente al otro $73 \%(n=8)$ que bajó de peso. En el grupo DOMUS se observó lo contrario, una mayoría $(62 \%, n=5)$ que ganó peso, y sólo $38 \%(n=3)$ logró reducirlo.

En cuanto al porcentaje de masa grasa, el grupo DOMUS redujo de media $1,7 \%$ de masa grasa (55\% disminuyó, 36\% aumentó y $9 \%$ se mantuvo), frente al 0,25\% de media que consiguió reducir el grupo AVANTE (el 62\% redujo y el $38 \%$ aumentó)

Al seleccionar de cada grupo (AVANTE y DOMUS) sólo aquellos que presentaban sobrepeso u obesidad al inicio del estudio y observamos la progresión de su IMC, obtuvimos que el grupo AVANTE consiguió mejores resultados. De 4

TABLA 2

Antropometría y nutrición.

Comparativa entre datos iniciales y finales $(n=19)$

Valor $\mathrm{P}$

Registro inicial [media $( \pm D E)$ Registro final [media $( \pm D E)$ ]

$\begin{array}{lc}\text { Peso }(\mathrm{kg}) & 71,4( \pm 20,0) \\ \text { IMC } & 25,8( \pm 4,6) \\ \text { \% Grasa corporal } & 28,3( \pm 10,8) \\ \text { Kcal } & 1868,5( \pm 364,0) \\ \text { \% Hidratos de carbono } & 42,2( \pm 4,9) \\ \text { \% Grasa total } & 38,9( \pm 4,4) \\ \text { \% Proteínas } & 19,1( \pm 2,5) \\ \text { Colesterol de la dieta (mg) } & 381,7( \pm 94,0)^{*} \\ \text { Fibra dietética }(\mathrm{g}) & 15,4( \pm 5,9) \\ \text { Índice KidMed } & 5,1( \pm 1,7)^{*}\end{array}$

$$
\begin{gathered}
70,4( \pm 18,9) \\
25,2( \pm 4,2) \\
27,4( \pm 9,8) \\
1758,1( \pm 352,5) \\
43,0( \pm 6,7) \\
39,4( \pm 6,6) \\
16,6( \pm 2,3) \\
299,8( \pm 63,0)^{*} \\
20,2( \pm 6,5) \\
7,1( \pm 1,3)^{*}
\end{gathered}
$$$$
{ }^{*} p<0,05
$$

Datos registrados al inicio y al final de la intervención. Datos expresados como Media ( \pm Desviación Estándar).

Macronutrientes expresados como el \% de Kcal totales que representan.

El Índice KidMed, permite valorar el grado de seguimiento de la Dieta Mediterránea con una puntuación de 0 a 12 (siendo el 0 una pobre adherencia y el 12 una buena adherencia). ${ }^{*} p<0,05$.

\section{TABLA 3}

Peso inicial y final de participantes con sobrepeso u obesidad.

Diferencia de peso en aquellos con sobrepeso u obesidad $(n=11)$

Registro inicial (IMC)

$\begin{array}{ll}\text { Paciente } 1 & 28,0 \\ \text { Paciente } 2 & 33,2 \\ \text { Paciente } 3 & 26,3 \\ \text { Paciente } 4 & 26,8 \\ \text { Paciente } 5 & 30,4 \\ \text { Paciente 6 } & 27,0 \\ \text { Paciente } 7 & 26,6 \\ \text { Paciente } 8 & 28,7 \\ \text { Paciente } 9 & 26,4 \\ \text { Paciente 10 } & 35,9 \\ \text { Paciente 11 } & 25,4 \\ \text { Total } & 28,6\end{array}$

Registro final (IMC)

Valor $\mathrm{P}$

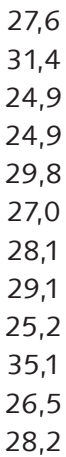

27,6

31,4

24,9

24,9

29,8

27,0

28,1

26,5

28,2

IMCs de los participantes con sobrepeso y obesidad registrados al inicio y al final de la intervención. 
pacientes que tenían sobrepeso, uno se mantuvo en sobrepeso y los otros dos bajaron a normopeso, y del cuarto paciente no se obtuvo medida final. De los 2 pacientes con obesidad, uno se mantuvo igual y el otro logró bajar a sobrepeso. En el grupo DOMUS en cambio, de los 4 pacientes que presentaban sobrepeso, uno se mantuvo igual y los otros dos aumentaron de peso permaneciendo en el rango de sobrepeso. Del cuarto paciente no se obtuvo medida final. El único paciente de este grupo clasificado como obeso, redujo peso hasta ser valorado como sobrepeso.

\section{DISCUSIÓN}

Los resultados del estudio están en consonancia con la tendencia a sobrepeso y obesidad en este tipo de población reflejada en un estudio de Bell AJ en 1992 (13) con una muestra de población de 183 individuos, en los que la prevalencia de obesidad estaba muy aumentada.

Con respecto al IMC, en nuestro estudio se obtuvo una media de 25,8, muy parecida a la obtenida por Melville et al. en 2008 (14) que fue 27,4 en sujetos con DI, suponiendo una clasificación como media de sobrepeso en nuestra muestra. En el estudio de Phillips KL et al. de 2014 (15), tanto la obesidad como la prevalencia de bajo peso fueron mayores entre los adolescentes con, que sin discapacidad de desarrollo (DD). Mikulovic J et al., en el 2014 (16), añadieron que el IMC medio de su muestra $(n=570)$ fue de $24,9 \mathrm{~kg} / \mathrm{m} 2 ; 45,6 \%$ de los participantes tenían exceso de peso (IMC $\geq 25)$, incluyendo $17,2 \%$ de obesos. Las mujeres presentaban mayor riesgo de sobrepeso que los hombres (53,9\% v/s 39,9\%) y obesidad (28,2\% v/s 9,5\%). En nuestro estudio, se observó lo contrario, las mujeres presentaron $38 \%$ de sobrepeso frente a $55 \%$ en hombres; y con respecto a la obesidad $0 \%$ en mujeres frente a $9 \%$ en los hombres.

Así lo reflejaron también Hsieh K et al. en 2013 (17): en comparación con la población general, los adultos ( $\geq 18$ años) con DI tenían mayor prevalencia de obesidad (38,3\% v/s 28\%) y obesidad mórbida. Ser mujer, tener síndrome de Down, tomar medicamentos que causan aumento de peso, realizar menor actividad física moderada, y beber mayor cantidad de refrescos se asociaron con mayores tasas de obesidad.Los adultos con DI, en general, tenían un alto riesgo de desarrollar obesidad, y las mujeres con DI tenían un alto riesgo de desarrollar obesidad mórbida.

En otros estudios, se comprobó que la prevalencia de obesidad era mayor en las personas que viven en sus entornos familiares y menor en los que viven en un hogar de grupo (18). Así, lo plasmaron también Doody CM y Doody O en 2012 (19): "Los grupos de pacientes con DI leve que residen en un hogar, ya sea grupal o en casa de la familia se encuentran en un mayor riesgo de obesidad y exceso de peso corporal que los de atención institucional". Los individuos de nuestro estudio pertenecen a hogares de grupo por lo que decidimos que era muy importante ofrecer un taller de técnicas culinarias, el cual fue muy bien aceptado y con el que aprendieron de una manera muy amena y divertida. Esto se apoyó en los datos, que ya se habían comprobado los efectos positivos de la técnica mediante la intervención llevada a cabo por Humphries $\mathrm{K}$ et al. en 2008 (20), consistente en un sistema de educación nutricional y directrices, planificación de menús y comidas, hacer la compra y cocinar, diseñado para las necesidades específicas de esta población.

La obesidad, es un importante problema de salud en la población con DI. Las causas son multifactoriales e incluyen conductas alimentarias inadecuadas, alta ingesta energética, tasa metabólica reducida, ejercicio reducido, hipotonía y alteraciones endocrinas (19). Entornos de vida menos restrictivos pueden estar asociados con una supervisión reducida y una mayor promoción de la elección individual (19), un componente que impacta en la malnutrición de estas personas (21), que puede resultar en un mayor acceso a la comida rápida (19), una exacerbación de la incapacidad que tienen para seleccionar sus alimentos de manera adecuada (21) y un menor énfasis en la actividad física (19).

Janet Robertson et al. (22) observaron, que adultos británicos con DI autoreportada tienen tasas más altas de obesidad (41,3\%, v/s 26,3\% de los sin DI), además de inactividad (25,3\%, v/s 10,7\%), consumo de tabaco y alcohol y pobre nutrición $(65,5 \%$ come fruta menos de 4 veces a la semana y $48,5 \%$ toma verdura menos de 4 veces a la semana). Estudios del Reino Unido y Australia verifican los datos anteriores, reportando una baja ingesta de frutas y verduras entre los adultos con DI. En nuestro caso, el consumo de fruta resultó disminuido al final del estudio con respecto al inicio del registro. También indicaron que una proporción significativa del mayor riesgo de obesidad, de consumo de tabaco y de pobre nutrición puede atribuirse a sus peores condiciones de vida (en lugar de a sus deficiencias intelectuales) (22).

Hay una estrecha relación entre la discapacidad y la mala salud pudiendo tener un mayor riesgo de desarrollar enfermedades crónicas (23) El estudio de la salud en relación con la edad de inicio de discapacidad puede iluminar el grado en que la discapacidad puede ser un factor de riesgo para el futuro de la mala salud (23). Dixon-Ibarra A y Horner-Johnson W (23), observaron que adultos con discapacidades tenían mayor probabilidad de tener : enfermedad coronaria, cáncer, diabetes, obesidad e hipertensión arterial. Las subpoblaciones de personas con discapacidad para toda la vida (es decir, física, mental, intelectual/desarrollo y sensorial) experimentaron un aumento similar de la probabilidad de sufrir dichas condiciones crónicas en comparación con las personas sin discapacidad. Los adultos con discapacidades, por tanto, eran más propensos a tener enfermedades crónicas que los adultos sin discapacidad, lo que indica que probablemente la discapacidad aumenta el riesgo de desarrollar problemas de salud.

En la muestra inicial se observó que la mayoría de los participantes no cubría la recomendación de ingesta de fibra dietética/día (25-30 g) (24), lo que está relacionado con una baja motilidad intestinal, aumento del riesgo de cáncer de colon, peores curvas de glucemia y su posible prevención para la resistencia a la insulina (diabetes tipo 2), aumento de la síntesis de colesterol endógeno y aumento, de enfermedad cardiovascular (25).Tras la intervención se observó un incremento de la ingesta de fibra $(p=0,004)$, lo que supondría una mejora en marcadores de riesgo para la salud.

Se comprobó que el consumo de frutas y verduras era bajo y por ello el de fibra también. En otro estudio (12) en el que, además de obtener resultados de una insuficiente ingesta de fruta y verdura, se comprobó que el nivel de actividad física fue bajo en todos los participantes $(87,5 \%$ no llegaban a las recomendaciones diarias). Robertson J et al. en 2014 (22), observaron que más del $80 \%$ de los adultos con DI caen en niveles de actividad física por bajo el nivel mínimo recomendado, similar con nuestra muestra.

Se obtuvo una media de 39\% de ingesta de lípidos antes de la intervención, coincidiendo con la de otros estudios (media de 38\% de lípidos) (26).

Según Donnelly JE et al. (27), las comidas pre-envasadas, con baja energía y grasa, pueden ser útiles para las PDI y de 
desarrollo, ya que simplifican la planificación de las comidas, limitan la elección de alimentos indeseables, enseñan los tamaños adecuados de las porciones, son convenientes y fáciles de preparar, y cuando se combinan con frutas y verduras proporcionan una comida de alta densidad nutricional y baja energía. Sin embargo, la muestra, al elegir alimentos más fáciles de preparar y técnicas más sencillas, recurrían a productos y técnicas que podrían aumentar su carga calórica e ingesta de grasa. Por ello se decidió incorporar en un taller de la intervención, aspectos relacionados con los mismos.

Para muchos adultos con DI, la hora de comer conlleva riesgos significativos para la salud. Se ha visto que el apoyo a la hora de comer es requerido por una minoría significativa de PDI, debido a dificultades motoras para alimentarse de forma autónoma y/o rechazo selectivo a los alimentos (28).

En esta población existen problemas psicomotores poco estudiados y arraigados, mitos acerca de que estas personas no pueden realizar actividad física de manera sistemática, aumentando con ello los problemas nutricionales (21).

Pett $M$ et al. (29) sometieron, a un grupo de jóvenes adultos a una intervención nutricional y de ejercicio; mientras sus padres se centraron en el modelado de los hábitos de vida saludables. Los adultos jóvenes mejoraron, después de la intervención, la presión arterial, el peso y el equilibrio. A los 3 meses de seguimiento, hubo reducciones generales de peso, presión arterial, circunferencia de cadera, y superaron las barreras del ejercicio. En el presente estudio se encontró una mejoría en la dieta en general (KidMed), concretamente de algunos nutrientes (colesterol, fibra y macronutrientes), y en la práctica regular de ejercicio físico.

La intervención en el comportamiento apoyada por los padres parece ser un complemento eficaz de una intervención de educación nutricional de 6 meses para lograr la pérdida de peso en adolescentes y adultos jóvenes con síndrome de Down (30). A los 6 meses, la media de peso corporal en el grupo de educación nutricional e intervención conductual (grupo 1) fue de 3,2 kg más bajo que en el grupo de educación nutricional (grupo 2).

Spanos D et al. (31), examinaron 22 estudios que informaron de la efectividad de intervenciones específicas destinadas a lograr la pérdida de peso en adultos con DI y obesidad.

Ningun estudio en este campo informó sobre una pérdida de peso clínicamente significativa del 5-10\% o 5-10 kg del peso corporal inicial (31). McCarran y Andrasik informaron de una reducción de peso estadísticamente significativa, de sólo 2,5 kg a las 14 semanas (31). Fox et al. (32) informaron de una pérdida de peso media de $3,4 \mathrm{~kg}$ en un grupo que contaba con la participación de los padres $y$, que fue significativamente diferente del grupo sin intervención paternal. McCarran y Andrasik encontraron una mayor, pero no significativa, diferencia entre la pérdida de peso de aquellos participantes que tenían a sus cuidadores implicados que los participantes que no los tenían (31). La pérdida de peso después de la intervención no se sostuvo con Fox et al., quienes informaron de la recuperación de peso a los tres y seis meses de seguimiento (31).

Fox et al. (32), concluyeron que la combinación de la actividad física y los enfoques conductuales podría conducir a una pérdida de peso superior al 5\% a las 10 semanas después de la intervención (31). Sin embargo, Fisher et al. (31) mostró que la incorporación de la actividad física no tuvo ningún efecto sobre la pérdida de peso. En nuestro caso, al separar a los participantes por centros, se observó que $50 \%$ del proyecto DOMUS mantuvieron su actividad física habitual, mientras que
50\% la incrementó. Los de grupo AVANTE, 64\% mantuvieron el ejercicio, 27\% lo aumentó y 9\% lo disminuyó, observándose además que los participantes del grupo AVANTE redujeron de media $2,3 \mathrm{~kg}$, frente a la ganancia media de $0,6 \mathrm{~kg}$ de peso del grupo DOMUS.

Los participantes, del estudio de Mikulovic J et al. (16), practicaban deporte en promedio $5,7 \mathrm{~h} /$ semana y eran sedentarios 21,8 h/semana. En sus resultados, mostraron que algunos hábitos de comportamiento se asociaron con una menor incidencia de sobrepeso y/u obesidad, como la práctica regular de deporte fuera de la institución, un buen cuidado personal del cuerpo o evitar el alcohol.

Mejoras bastante modestas se observaron en la mayoría de las intervenciones, basadas en el ejercicio, en la nutrición, o en ambas, o globales en hábitos saludables, y que suponen cambios de hábitos que no modifican marcadores de salud de forma rápida (grasa corporal, IMC, glucosa o colesterol en sangre). Hinckson EA et al., (33), determinaron la eficacia de un programa de 10 semanas en el manejo de peso, a través de cambios en la actividad física y los comportamientos de nutrición en los niños y jóvenes con DI y sobrepeso u obesidad en Nueva Zelanda. El programa consistió en 18 sesiones centradas en la actividad física y la nutrición. Los cambios se midieron inmediatamente después de finalizar el programa y a las 24 semanas. Se observó que el único cambio producido fue en el test-caminata de seis minutos donde los participantes a las 24 semanas caminaron 51 metros más. Sin embargo, hubo una reducción sustancial en el consumo de productos de confitería y chocolate en los dos puntos de medición. Los padres comentaron que durante el programa hubo menos visitas al hospital y ausencias a la escuela relacionadas con la enfermedad.

Intervenciones más consistentes, no sólo basadas en la educación de hábitos saludables, han reportado mejoras más significativas como las de diversos autores que figuran en el estudio de Curtin C et al. de 2013 (31), que realizaron dietas o entrenamientos personalizados.

Las intervenciones multifactoriales, definidas como "una combinación de dieta y actividad física con una estrategia de cambio de comportamiento para influir sobre el estilo de vida", todas reportaron una disminución en el peso corporal, o IMC, pero parece que la mayor pérdida de peso fue por las intervenciones que recomiendan dietas con déficit de energía (31).

En conclusión, la alimentación de este grupo de personas era inadecuada. Con una serie de talleres de educación nutricional se pudo mejorar en algunos aspectos, comprobando que son una herramienta útil para trabajar con este colectivo consiguiendo cambios significativos. El ejercicio físico representó una mejora de los marcadores del estudio. Es un colectivo que se va a beneficiar de la educación nutricional tanto para prevenir la obesidad y corregir errores, como para facilitar la vida independiente y autónoma, así como mejorar su estado de salud y fisiológico. Hacen falta más estudios que continúen la línea de investigación en este colectivo.

Las medidas corporales no se pudieron medir en unas condiciones óptimas, según los estándares establecidos para estos equipos de BIA, a pesar de facilitar un protocolo de referencia, pero no se realizaron en condiciones estandarizadas de ayuno e hidratación óptimas. Esto resta validez a estos parámetros, aunque sirven de orientación.

El cuestionario KidMed, está validado para edades comprendidas entre 2-24 años, y no está validado para esta población. Sin embargo nos permitió tener una referencia global de ciertos hábitos antes y después de la intervención, 
así como ciertos patrones independientes que recoge este cuestionario y que son igualmente interesantes.

\section{LIMITACIONES}

Se trata de un estudio descriptivo, del que no se pueden establecer causalidades. La muestra fue incidental y el tamaño muestral no fue calculado para observar ningún efecto determinado.

La muestra fue heterogénea en cuanto a los tipos de discapacidades que presentaban los sujetos, lo que no evita posibles diferencias inter-sujetos en la respuesta a la intervención.

La antropometría se midió en las mejores condiciones posibles, pero no en las indoneas según el fabricante.

Aunque los registros de alimentos y comidas fueron recogidos de forma prospectiva, suponen un compromiso de cada sujeto y/o su tutor/monitor en cumplimentar y detallar todo, lo cual fue complicado verificar.

\section{RESUMEN}

Las personas discapacidad intelectual (PDI) (0,7-1,5\% de la población) presentan mayores tasas de obesidad, pues los valores calóricos y estado nutricional son deficientes. Se quiso conocer los hábitos nutricionales, analizar la eficacia de la educación nutricional y el efecto de mejora con talleres educacionales, en PDI. Se realizó una valoración nutricional, antropométrica y clínica a 19 usuarios. Se diseñó una encuesta Ad Hoc sobre hábitos deportivos, historia y dietética, registro alimentario (2 días laborales, 1 fin de semana), y cuestionario Adherencia a Dieta Mediterránea. Los talleres de ejercicio y nutrición contaron con explicación teórica, práctica y juegos. La muestra $(n=19)$, de edad media 31 $( \pm 5,9)$ años, 11 hombres, 8 mujeres, presentaron un $42 \%$ sobrepeso y un $16 \%$ obesidad. El $11 \%$ presentaba perímetro de cintura de riesgo. Ninguno cumplía con las recomendaciones de ejercicio diario. El 100\% presentó una ingesta nutricional inadecuada. La educación nutricional y ejercicio físico son herramientas útiles en este colectivo, consiguiendo mejoras significativas.

Palabras clave: Discapacidad intelectual; educación nutricional; obesidad; ejercicio físico; hábitos.

Agradecimientos: A los participantes del mismo y a las asociaciones involucradas (Fundación Síndrome de Down y Avante3), ya que sin su ayuda no hubiese sido posible esta investigacion.

Conflicto de intereses: No existió ningún conflicto de interés por parte de ninguno de los autores.

\section{BIBLIOGRAFÍA}

1. Martínez-Leal R. Salvador-Carulla L. Ruiz Gutiérrez-Colosía M. Nadal M. Novell-Alsina R. Martorell A. et al. Health in people with intellectual disabilities in Spain: European study POMONA-II, Rev Neurol. 2011; 53:406-14.

2. Verdugo MA. Analysis of the definition of intellectual disability of the American Association on Mental Retardation in 2002, Rev Española Discapacidad Intelectual 2002; 34: 1-17.

3. Bodde AE. Seo DC. Frey GC. Van Puymbroeck M. Lohrmann $D K$. The effect of a designed health education intervention on physical activity knowledge and participation of adults with intellectual disabilities, Am J Health Promot. 2012; 26:313-6.

4. Discapacidadonline. Down syndrome and hypotonic psychomotor intervention. [Fecha de consulta: Julio 6 del 2014]. Disponible en: http://www.discapacidadonline. com/sindrome-down-hipotonia-intervencion-psicomotricidad.html

5. Saravia L. Food in people with Down syndrome. Centro de postgrado. [Fecha de consulta: Julio 6 del 2014]. Disponible en: http://www.cdp.edu.uy/blog/2011/02/alimentacionen-personas-con-sindrome-de-down/

6. Soler A. Nutritional intervention methodology in a group of people with Down síndrome in Murcia. Murcia: Universidad Católica San Antonio; 2005.

7. Grondhuis SN. Aman MG. Overweight and obesity in youth with developmental disabilities: a call to action, J Intellect Disabil Res. 2013; 58:787-99.

8. Jankowicz-Szymanska A. Mikolajczyk E. Wojtanowski W. The effect of the degree of disability on nutritional status and flat feet in adolescents with Down syndrome, Res Dev Disabil. 2013; 34:3686-90.

9. Heller T. McCubbin JA. Drum C. Peterson J. Physical activity and nutrition health promotion interventions: what is working for people with intellectual disabilities?, Intellect Dev Disabil. 2011; 49:26-36.

10. Serra-Majem L. Ribas L. Ngo J. Ortega RM. García A. Pérez-Rodrigo C. et al. Food, youth and the Mediterranean diet in Spain. Development of KIDMED, Mediterranean Diet Quality Index in children and adolescents, Public Health Nutr. 2004; 7: 931-35.

11. Salas-Salvadó J. Rubio MA. Barbany M. Moreno B y grupo colaborativo SEEDO. SEEDO 2007 consensus for the evaluation of overweight and obesity and the establishment of therapeutic intervention criteria, Med Clin (Barc) 2007; 128:184-96.

12. Adolfsson P. Mattsson-Sydner Y. Fjellström C. Lewin B. Andersson $A$. Observed dietary intake in adults with intellectual disability living in the community, Food Nutr Res. 2008; 52. http://dx.doi.org/10.3402/fnr.v52i0.1857.

13. Bell AJ. Bhate MS. Prevalence of overweight and obesity in Down's syndrome and other mentally handicapped adults living in the community, J Intellect Disabil Res 1992; 36:359-64.

14. Melville CA. Cooper SA. McGrother CW. Thorp CF. Collacott $R$. Obesity in adults with Down sindrome: a case-control study, J Intellectual Disability Res 2005; 49:125-33.

15. Phillips KL. Schieve LA. Visser S. Boulet S. Sharma AJ. Kogan MD. et al. Prevalence and impact of unhealthy weight in a national sample of US adolescents with autism and other learning and behavioral disabilities, Matern Child Health J. 2014; 18:1964-75.

16. Mikulovic J. Vanhelst J. Salleron J. Marcellini A. Compte R. Fardy PS. et al. Overweight in intellectually-disabled population: physical, behavioral and psychological characteristics, Res Dev Disabil. 2014; 35:153-61.

17. Hsieh K. Rimmer JH. Heller T. Obesity and associated factors in adults with intellectual disability, J Intellect Disabil Res. 2014; 58:851-63.

18. Rubin SS. Rimmer JH. Chicoine B. Braddock D. McGuire DE. Overweight prevalence in persons with Down syndrome, Ment Retard. 1998; 36:175-81.

19. Doody CM. Doody O. Health promotion for people with intellectual disability and obesity, Br J Nurs. 2012; 21:460, 462-5.

20. Humphries K. Traci MA. Seekins T. Nutrition education and support program for community-dwelling adults with intellectual disabilities. Intellect Dev Disabil. 2008; 46:335-45.

21. Ramos-Jiménez A. Wall-Medrano A. Hernández-Torres RP. Physiological and social factors associated with body mass 
of young Mexicans with intellectual disabilities, Nutr Hosp 2012; 27:2020-7.

22. Dixon-Ibarra A. Horner-Johnson W. Disability status as an antecedent to chronic conditions: National Health Interview Survey, 2006-2012, Prev Chronic Dis 2014; http://dx.doi. org/10.5888/pcd11.130251.

23. Escudero-Álvarez E. González-Sánchez P. Dietary fibre, Nutr Hosp. 2006; 21: 61-72.

24. Plaza-Díaz J. Gil A. I Jornada Universitaria UCM-CLAS sobre Fibra Dietética, Fibra y microbiota intestinal 2012; p 12-21.

25. Robertson J. Emerson E. Baines S. Hatton C. Obesity and health behaviours of British adults with self-reported intellectual impairments: cross sectional survey, BMC Public Health 2014; 14:219.

26. Soler A. Xandri JM. Nutritional status of intellectual disabled persons with Down syndrome, Nutr Hosp. 2011; 26:1059-66.

27. Donnelly JE. Saunders RR. Saunders M. Washburn RA. Sullivan DK. Gibson CA. et al. Weight management for individuals with intellectual and developmental disabilities: rationale and design for an 18 month randomized trial, Contemp Clin Trials 2013; 36:116-24.
28. Ball SL. Panter SG. Redley M. Proctor CA. Byrne K. Clare IC. et al. The extent and nature of need for mealtime support among adults with intellectual disabilities, J Intellect Disabil Res. 2012; 56:382-401.

29. Pett M. Clark L. Eldredge A. Cardell B. Jordan K. Chambless C. Burley J. Effecting healthy lifestyle changes in overweight and obese young adults with intellectual disability, Am J Intellect Dev Disabil. 2013; 118:224-43.

30. Curtin C. Bandini LG. Must A. Gleason J. Lividini K. Phillips S. et al. Parent support improves weight loss in adolescents and young adults with Down syndrome, J Pediatr. 2013; 163:1402-8.

31. Spanos D. Melville CA. Hankey CR. Weight management interventions in adults with intellectual disabilities and obesity: a systematic review of the evidence, Nutr. J 2013; 12:132.

32. Fox RA. Rosenberg R. Rotatori AF. Parent involvement in a treatment program for obese retarded adults, J Behav Ther Exp Psychiat. 1985; 16:45-8.

33. Hinckson EA. Dickinson A. Water T. Sands M. Penman L. Physical activity, dietary habits and overall health in overweight and obese children and youth with intellectual disability or autism, Res Dev Disabil. 2013; 34:1170-8. 